\title{
Recent Advances in Modeling Fission Cross Sections over Intermediate Structures
}

\author{
Olivier Bouland ${ }^{1,2, a}$, J. Eric Lynn ${ }^{1}$, and Patrick Talou ${ }^{1}$ \\ 1 T-2 - Nuclear and Particle Physics, Astrophysics and Cosmology Group, Theoretical Division, Los Alamos National \\ Laboratory, Los Alamos, NM 87545, USA. \\ 2 Laboratoire d'Etudes de Physique, DEN/DER/SPRC, Commisariat à l'Energie Atomique, 13108 St-Paul-lez-Durance, \\ C.E. Cadarache - France.
}

\begin{abstract}
More accurate fission cross section calculations in presence of underlying intermediate structure are strongly desired. This paper recalls the common approximations used below the fission threshold and quantifies their impact. In particular, an exact expanded R-matrix Monte Carlo calculation of the intermediate structure, deeply mixed with the fluctuations of the class-I and II decay amplitudes, is shown. This paper also insists on the microscopic structure of the level densities as a function of the nucleus deformation and show preliminary neutron induced fission cross section calculations for ${ }^{239} \mathrm{Pu}$ and ${ }^{240} \mathrm{Pu}$ using newly calculated combinatorial level densities. Comparisons with recent evaluated and measured fission cross sections are made.
\end{abstract}

\section{Introduction}

The modelling of fission cross sections over intermediate structure has been challenging since the first experimental discoveries in 1968 of the phenomenon of narrow intermediate structure in slow neutron fission measurements on ${ }^{237} \mathrm{~Np}$ and ${ }^{240} \mathrm{Pu}$ nuclei (respectively by Paya et al. [1] and Migneco et al. [2]). This phenomenon, explained by the coupling between highly deformed vibrational classII states (located in a secondary well of the nucleus potential energy) and the class-I states at ground-state deformation, has required modifications to the R-matrix formalism for interactions in the deformation channels. This extended R-matrix reaction theory involving the splitting of the reaction Hamiltonian into deformation, intrinsic and coupling terms was developed in particular by Lynn [3], and led to various asymptotic formulations of the average fission cross section depending of the strength of the coupling element. Ever increasing computing capabilities have made possible more exact calculations of the average fission cross section, using a Monte Carlo sampling of energies, fission and coupling widths of the class-II states as well as the characteristics of the class-I states from the statistical distribution of levels and partial widths. A few attempts were made in the past $[4,5]$, but the most significant application of this method was performed in 2002 by Lynn and Hayes [6] over a wide range of actinide fission cross sections. In addition to better predict the magnitude of the cross section, this Monte Carlo approach paves the way for modeling genuine fluctuations in the Unresolved Resonance energy Range (at the right energies and with correct fluctuation envelopes) and provides a more

\footnotetext{
a e-mail: obouland@lanl.gov or olivier.bouland @cea.fr
}

fundamental background for evaluating meaningful fission width parameters in the Resolved Resonance Range. The present work aims at performing accurate fission cross section calculations on the basis of sound physics (less phenomenology, fitted parameters and dependency on experimental fits) in order to

- better model current applications but also better predict cross sections for difficult or impossible to measure nuclei,

- better estimate of related fission quantities (fission fragments, fission spectra, etc.),

- reinforce our confidence in the estimation of parameter uncertainties and associated covariance data.

This paper will summarize our current advances on calculated cross sections and illustrate them by preliminary results on neutron induced reactions on ${ }^{239} \mathrm{Pu}$ and ${ }^{240} \mathrm{Pu}$ with comparison to recent evaluations and fission data. Finally foreseen developments will be discussed.

\section{Theoretical Background}

\subsection{Classic approaches in resonance range analyses}

Low energy cross sections are well reproduced by classic $R$-matrix theory of nuclear reactions, which is based on the eigenfunctions and eigenvalues of the full nuclear Hamiltonian in an internal region with boundary conditions imposed to the reaction channels at the region surface. These conditions connect the external wave functions to the $R$ matrix internal states and so define an ensemble of eigenfunctions in the inner region. It also introduces the concept of reaction channel defining the maximum of radial

This is an Open Access article distributed under the terms of the Creative Commons Attribution-Noncommercial License, which permits unrestricted use, distribution, and reproduction in any noncommercial medium, provided the original work is properly cited. 
distance within which a nuclear force-type interaction between the two "bodies" involved may occur. An entrance or an exit reaction channel is defined by its quantum numbers $\alpha, l, s, J$ which qualify respectively the nature and the number of nucleons of each component of the pair $(\alpha)$, the relative orbital angular momentum of the moving particletarget (or residual) nucleus pair $(l)$, the channel spin $(s)$ and the total angular momentum $(J)$ of the system.

In the original R-matrix theory fission channels are assumed to be treated as true particle reaction channels, as just described, with the channel radii close to the saddle point. We refer to these as the fission product channels, each of which is denoted by the masses of the pair of products, their states of excitation, their angular momenta and parities, etc. Bohr [7] pointed out, however, that in a physical sense a fission channel should be regarded as being entered at the saddle point, where the deforming nucleus is relatively "cold", and a specific channel would be defined by the internal state of excitation of the deforming nucleus. It appeared that some of the properties of this internal excitation, notably the $K$ quantum number (i.e.; the projection of $J$ on the elongation axis), could be "frozen in" already at the saddle point. This point of view has since dominated the analysis of cross sections such as Reich-Moore, in which only a few fission channels are stipulated. The reduced width amplitudes of the fission product channels can be regarded as being correlated with the amplitude of the channel at the fission barrier, thus justifying the use of the barrier channel (or, "transition state") concept (illustrated in Fig. 1) in R-matrix theory. These channels require for their description a parameter $\eta$ characterizing globally the deformation and a wave function $\varphi(\eta)$ which satisfies the Schrodinger equation with suitable boundary condition at the channel entrance close to the saddle point deformation:

$$
-\frac{\hbar^{2}}{2 B} \frac{\partial^{2} \varphi(\eta)}{\partial \eta}+(v(\eta)-E) \varphi(\eta)=0,
$$

with $B$ the inertial parameter, including the effect of nuclear interactions among the "internal" degrees of freedom as the deformation changes.

In the Unresolved Resonance energy Range on which statistics applies, Hauser-Feschbach (HF) theory with width fluctuations, averaging $R$-matrix theory over the Gaussian orthogonal ensemble, is appropriately used for fission cross section measurement analyses. However HF theory is often associated with effective single hump barrier penetrabilities for many fissile nuclides studies (such as those constrained by the current status of the SAMMY code). This approach allows a satisfactory reproduction of average fission cross sections but relies heavily on the adjustment of phenomenological average fission widths, fission barrier heights.

\subsection{Inclusion of Fission in Classic $R$-matrix Theory}

Experimental evidence of intermediate states and doublehumped barrier has motivated the inclusion of fission deformation modes in $R$-matrix theory in a more formal way.

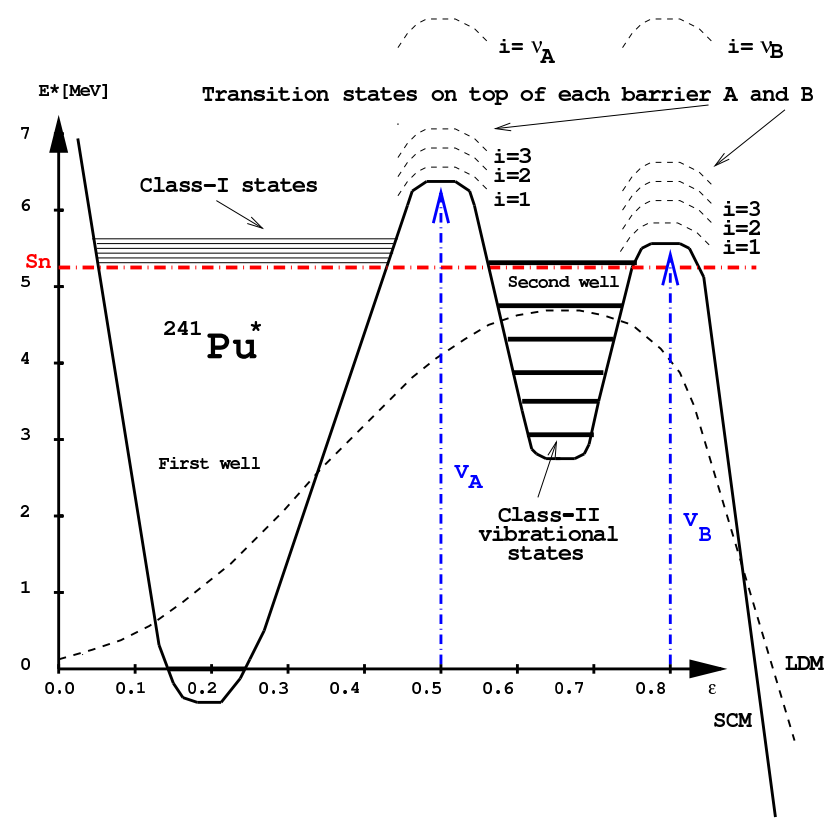

Fig. 1. Potential energy of deformation of the ${ }^{241} \mathrm{Pu}^{*}$ system as a function of the elongation. Comparison between the single hump of the Liquid Drop Model (LDM) and the the double-hump barrier shape of the Shell Correction Method (SCM).

A generalized variable $\eta$, which describes the overall continuous elongation of the system along the fission path, was introduced by Lynn [3]. The Hamiltonian operator:

$$
H=H_{\eta}+H_{\text {int }}\left(\zeta, \eta_{0}\right)+H_{c}\left(\eta, \zeta ; \eta_{0}\right),
$$

is decomposed into

- a collective component, $H_{\eta}$, depending explicitly on the deformation parameter,

- an intrinsic Hamiltonian component, $H_{i n t}$, that depends on the individual nucleon coordinates, $\zeta$, and,

- a coupling Hamiltonian, $H_{c}$.

The eigenfunctions and eigenvalues of $H_{\text {int }}$ and $H_{\eta}$ are respectively $\chi_{\mu}(\eta)$ and $\varepsilon_{\mu}(\eta) \equiv \epsilon_{\mu}(\eta)-\epsilon_{\mu}\left(\eta_{0}\right)$; the intrinsic excitation energies relative to "ground state" and, $\phi_{\nu}(\eta)$ and $\varepsilon_{v}$. It is convenient to expand the eigenstates $X_{\lambda}$ of the generalized R-matrix of the internal region in terms of product pairs of the quasi-vibrational functions $\phi_{\nu}(\eta)$ and the intrinsic functions $\chi_{\mu}(\eta)$ defined at some special deformations $\eta_{0}$ such as those of the normal and meta-stable values of the double hump fission barrier. These can be constituted of two groups of auxiliary states $\left(X_{\lambda_{I}}^{I}\right.$ and $\left.X_{\lambda_{I}}^{I I}\right)$ formed from expansions limited to vibrational states localized principally in either the primary or secondary well of the deformation potential $v(\eta)$ :

$$
X_{\lambda}^{I}=\sum_{\nu \mu} C_{\lambda(\nu \mu)} \phi_{\nu(\mu)}^{I} \chi_{\mu},
$$

for the class-I states, and similarly for the class-II states.

The final $R$-matrix compound nucleus states $X_{\lambda}$, which can be classed asymptotically as either normal (class-I) or meta-stable well (class-II) states, contain a mixture of 
these two sets of auxiliary states (expressed by the admixture coefficients $C_{\lambda(v \mu)}$ into eigenstates $\lambda$ ). If the intermediate barrier between the two wells is high relative to the excitation energy the overlap between class-I and -II vibrational states is small and the mixing of the two classes is weak, and the class-II compound states (with relatively high vibrational amplitudes at the outer barrier) then appear as clear intermediate resonances.

\section{Treatment of class-I and class-II states coupling.}

For a given class-II state, $\lambda_{I I}$, the average over neighboring class-I levels of the squared matrix elements between it and the class-I states, $H_{c}^{2}\left(\lambda_{I I} \lambda_{I}\right)$, is proportional to the mean coupling width, $\left\langle\Gamma_{\lambda_{I I}(c)}\right\rangle$ :

$$
\left\langle H_{c}^{2}\left(\lambda_{I I} \lambda_{I}\right)\right\rangle_{\alpha}=\left\langle\Gamma_{\lambda_{I I}(c)}\right\rangle_{\alpha} D_{I} / 2 \pi,
$$

with $\left\langle\Gamma_{\lambda_{I I}(c)}\right\rangle_{\alpha}$ being deduced from the transmission coefficient $T_{A}$ across an inner barrier channel $\alpha$ :

$$
2 \pi\left\langle\Gamma_{\lambda_{I I}(c)}\right\rangle_{\alpha} / D_{I I}=T_{A}(\alpha) .
$$

Similarly, the partial fission component of the average classII fission width across the $\beta$ outer barrier channels is deduced from the transmission coefficient $T_{B}$

$$
2 \pi\left\langle\Gamma_{\lambda_{I I}(f)}\right\rangle_{\beta} / D_{I I}=T_{B}(\beta) .
$$

Determination of $E_{\lambda}$ and $X_{\lambda}$ at sub-barrier energies. In the sub-barrier case, the assumption that the total fission width of a class-II state is always significantly less than the class-II level spacing, is reasonable. According to Ref. [3], in the most general case where the total fission width of the class-II state considered is smaller than a few class-I mean spacings (e.g.; $\Gamma_{\lambda_{I I}}<4 D_{I}$ ), an exact diagonalization of the single class-II state considered (the contributions of the distant class-II are added later) with its discrete classI neighbors must be performed to calculate the eigenvalues, $E_{\lambda}$, of the Hamiltonian, $H_{c}$ (Eq. 2), in the intermediate structure. The coefficients $C_{\lambda\left(\lambda_{I}\right)}$ and $C_{\lambda\left(\lambda_{I I}\right)}$ are then:

$$
C_{\lambda\left(\lambda_{I}\right)}=-\frac{\left\langle H_{c}^{2}\left(\lambda_{I I} \lambda_{I}\right)\right\rangle}{E_{\lambda_{I}}-E_{\lambda}} C_{\lambda\left(\lambda_{I I}\right)}
$$

and,

$$
C_{\lambda\left(\lambda_{I I}\right)}^{2}=\left[\sum_{\lambda_{I}} \frac{\left|\left\langle H_{c}\left(\lambda_{I I} \lambda_{I}\right)\right\rangle\right|^{2}}{\left(E_{\lambda_{I}}-E_{\lambda}\right)^{2}}+1\right]^{-1} .
$$

$C_{\lambda\left(\lambda_{I}\right)}$ and $C_{\lambda\left(\lambda_{I I}\right)}$ are used to calculate the partial resonance parameters corresponding to open reactions in both wells. In particular the R-matrix fission width, entering in the average cross section formula in narrow resonance approximation, is calculated as:

$$
\Gamma_{\lambda}(f)=2 P_{f} \sum_{\nu=1}^{v_{\text {outer }}^{\text {max }}} C_{\lambda\left(\lambda_{I I}\right)}^{2} \times \gamma_{\lambda_{I I}, v}^{2}(f)
$$

with $v_{\text {outer }}^{\max }$ being the maximum number of outer barrier fission channels open or partially open, $P_{f}$, the barrier penetration factor from beyond the outer saddle point and, $\gamma_{\lambda_{I I}, v}$, the class-II decay width amplitude by fission into an outer channel $v$.

\section{Construction of Individual and Continuum States}

\subsection{Individual compound nucleus and transition states}

In the case of an even-even target (or fissioning) nucleus, only collective states exist at low energies. The existence of a two quasi-particle (2qp) intrinsic state is correlated to the breaking of the pairing energy force between two neutrons or two protons and its excited energy is the sum of the two independent quasi-particle energies:

$$
\begin{aligned}
E_{2 q p}^{e x c} & =E_{v}+E_{v^{\prime}} \\
& =\sqrt{\left(e_{v}-\lambda_{n / p}\right)^{2}+\Delta_{n / p}^{2}}+\sqrt{\left(e_{v^{\prime}}-\lambda_{n / p}\right)^{2}+\Delta_{n / p}^{2}(10)}
\end{aligned}
$$

with $e_{v}$ and $\lambda_{n / p}$ being respectively the single-particle energy and the Fermi energy. Associated spin and parity are respectively:

$$
K_{2 q p}=\left|K_{v} \pm K_{v^{\prime}}\right|, \quad \pi_{2 q p}=\pi_{v} * \pi_{v^{\prime}} .
$$

This implies that only individual state band heads (on top of which rotational bands are built) of collective nature exist at low energies:

$$
E_{\text {rot }}=\left\{h^{2} /(2 \mathfrak{J})\right\}\{J(J+1)-K(K+1)\}
$$

with,

$$
\begin{aligned}
& J^{\pi}=K^{\pi},(K+1)^{\pi},(K+2)^{\pi}, \\
& =0^{+}, 2^{+}, 4^{+} \text {, } \\
& =1^{-}, 3^{-}, 5^{-} \text {, } \\
& \text { for } K \neq 0 \\
& \text { for } K=0^{+} \\
& \text {for } K=0^{-}
\end{aligned}
$$

Equation 10 clearly depicts the minimum energy gap value, two pairing energies $\left(2 \Delta_{n / p}\right)$, encountered between the vibrational ground state and the lowest quasi-particle state. This picture is more complicated for odd-mass $\left(E_{1 q p}^{e x c}=\right.$ $\left.E_{n / p}\right)$ and odd-odd nuclei $\left(E_{2 \times 1 q p}^{e x c}=E_{n}+E_{p}\right)$ which present respectively 1 neutron or proton quasi-particle state excitation and, 1 neutron and 1 proton quasi-particle state excitations in the ground state. The construction of the corresponding individual level sequence requires, prior to rotational enhancement building, a merging of the low energy vibrational and simple quasi-particle excitations to form combinatorial state band heads of energy, spin and parity:

$$
\begin{aligned}
E_{N}^{e x c} & =E_{q p}^{e x c}+E_{v i b}^{e x c} \\
K_{N} & =\left|K_{q p} \pm K_{v i b}\right| \\
\pi_{N} & =\pi_{q p} * \pi_{v i b}
\end{aligned}
$$

Low-lying collective levels are usually known experimentally up to about $1 \mathrm{MeV}$. The ${ }^{240} \mathrm{Pu}$ vibrational inelastic levels [8] include a mass asymmetry vibration with $K^{\pi}=1^{-}$at $E^{*}=0.60 \mathrm{MeV}$, a beta-vibration, $K^{\pi}=0^{+}$at $E^{*}=0.86 \mathrm{MeV}$, a bending-vibration, $K^{\pi}=1^{-}$at $E^{*}=0.94$ $\mathrm{MeV}$ and a gamma vibration, $K^{\pi}=2^{+}$at $E^{*}=1.14 \mathrm{MeV}$. Above this energy, extra unobserved levels $\left(J^{\pi}=5^{+}, E^{*}=\right.$ 1.145 and $1.298 \mathrm{MeV} ; J^{\pi}=6^{+}, E^{*}=1.223$ and 1.316 $\mathrm{MeV} ; J^{\pi}=4^{-}, E^{*}=1.334 \mathrm{MeV} ; J^{\pi}=5^{-}, E^{*}=1.399$ 


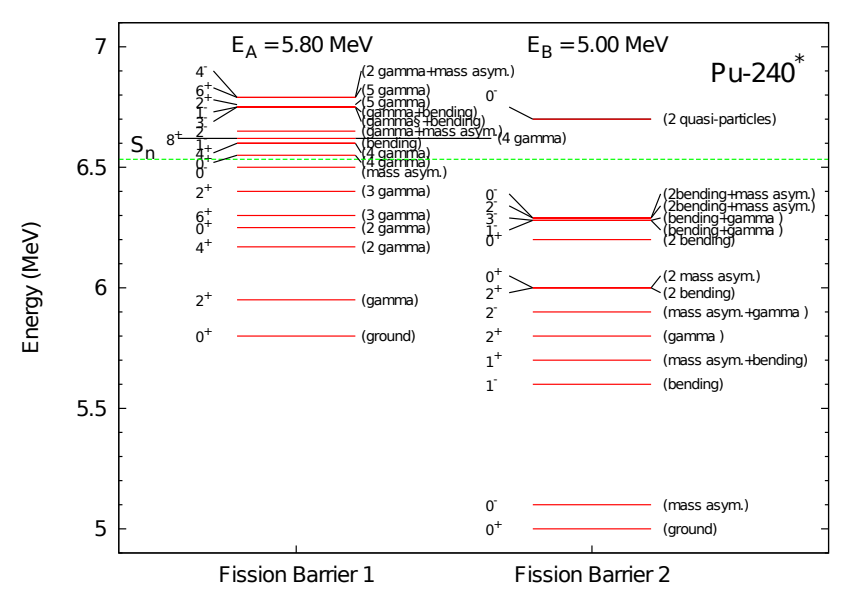

Fig. 2. Schematic of the ${ }^{240} \mathrm{Pu}^{*}$ individual low-lying vibrational sequence used at the inner and outer barriers.

$\mathrm{MeV} ; J^{\pi}=6^{-}, E^{*}=1.387$ and $1.477 \mathrm{MeV}$ were added to ensure the consistency with the combinatorial level density used above this individual state sequence. For an odd-n mass target nucleus such as ${ }^{239} \mathrm{Pu}$, the inelastic spectrum also includes low energy single-particle neutron states [9]. The lowest single-particle levels for ${ }^{239} \mathrm{Pu}$ target nucleus are respectively in $\left[N, n_{z}, \Lambda, \Omega\right]$ asymptotic quantum numbers notation $1 / 2^{+}[631]$ at $E^{*}=7.8 \mathrm{keV} ; 5 / 2^{+}[622]$ at $E^{*}=285 \mathrm{keV}, 7 / 2^{-}[743]$ at $E^{*}=392 \mathrm{keV}, 1 / 2^{-}[631]^{*} 0^{-}$ at $E^{*}=470 \mathrm{keV}$, and $7 / 2^{+}[624]$ at $E^{*}=512 \mathrm{keV}$.

A similar approach is adopted to construct the transition states on top of each barrier with the average level spacing adjusted to the deformation. A schematic of the individual low-lying vibrational sequence used for the inner barrier of the ${ }^{240} \mathrm{Pu}^{*}$ is shown in Fig. 2 (left-hand side) which mostly exhibits combinations of gamma vibrations. The lowest single particle neutron state (2qp) appears at $1.39 \mathrm{MeV}$ above the inner barrier, and so is not visible on this picture. The right-hand side of Fig. 2 shows a similar schematic for the outer barrier but exhibits at low energy a mass asymmetry vibration and at rather higher energy a bending vibration and then, simple combinations of them. The lowest single particle neutron state (2qp) shows up at 1.7 MeV above the outer barrier.

\subsection{Construction of level densities}

A combinatorial procedure was adopted to construct level densities as a function of the excitation energy and the nucleus deformation. This procedure is a generalization of the method used in section 3.1 for creating the sequence of individual states. It deals now with multi-combinations of neutron and/or proton quasi-particle states (leading to 1qp, 3qp, 5qp, etc. or 2qp, 4qp, 6qp, etc. states) and multicombinations of mass asymmetry, bending, gamma, etc. vibrational states. Subsequent combinations of these multiquasi-particle and multi-vibrational states are made, on the model of Eqs. 13 to form the combinatorial rotational band heads.

The main ingredients of this procedure are the pairing gaps, the moment of inertia $h^{2} /(2 \mathfrak{J})$ and, the vibrational

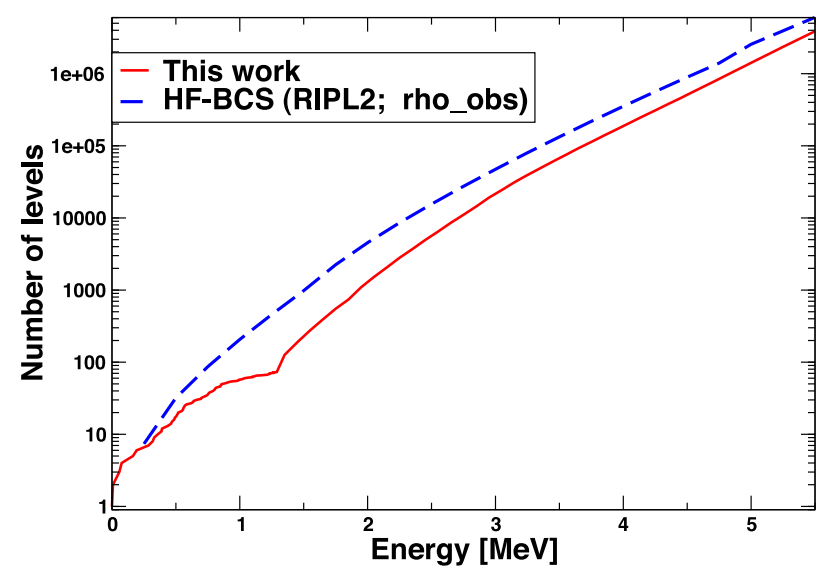

Fig. 3. Cumulated inelastic level densities (Total level density integrated over the energy spectrum from $0 \mathrm{eV}$ to the considered excitation energy $E_{\text {exc }}$ ) of the ${ }^{239} \mathrm{Pu}$ target nucleus as a function of $E_{\text {exc }}$. Our calculation (solid curve) is compared to the HF-BCS prediction (dashed-curve) tabulated in the RIPL-2 database. The plot displays at low energy the individual level density, matching the continuum level density calculation at $1.3 \mathrm{MeV}$, used in our calculation.

and the single-particle state sequences at barrier deformations. The ${ }^{240} \mathrm{Pu}^{*}$ single-particle neutron and proton states at the inner deformation are currently extracted from Nilsson et al. [10] (valid for the first hump and secondary minimum only) and the states sequences at the outer deformation are supplied by Möller et al. [11] slightly extrapolated for an additional degree of liberty in the shape of the nucleus. Another sensitive parameter, as energy increases, is the so-called blocking attenuation parameter $\left(b_{n / p}\right)$. This later insures the gradual collapse of the nucleon pairing gap $\left(\Delta_{n / p}\right)$ with energy because of the decrease of the number of unoccupied single-particle states below the Fermi energy:

$$
\Delta_{n / p}^{\prime}=\Delta_{n / p} \exp \left[-q_{n / p, e f f}^{2} / b_{n / p}^{2}\right],
$$

with $q_{n / p, e f f}$, the effective number of quasi-particles in a state following Gaussian distribution. This leads to a lowering of the ground state excitation energy value $\left(E_{N}\right)$ with energy of the combinatorial level $N$ :

$$
E_{N}^{\prime}=E_{N}-\frac{1}{4} \sum_{x, p} \rho_{x S}\left[\Delta_{x}^{2}-\Delta_{x}^{\prime 2}\right]
$$

with $\rho_{x S}$, the nucleon single-particle state densities.

Table 1. Fundamental barrier values $[\mathrm{MeV}]$ used for the ${ }^{240} \mathrm{Pu}^{*}$ compound nucleus in this preliminary work compared with data from HF-BCS predictions [12] and macroscopic-microscopic Finite Range Liquid Drop Model calculations [13].

\begin{tabular}{c|ccc}
\hline Barrier & $\begin{array}{c}\text { This } \\
\text { work }\end{array}$ & $\begin{array}{c}\text { HF-BCS } \\
{[12]}\end{array}$ & $\begin{array}{c}\text { FRLDM } \\
{[13]}\end{array}$ \\
\hline Inner & 5.8 & 6.5 & 5.99 \\
Outer & 5.0 & 5.61 & 4.91 \\
\hline
\end{tabular}


Figure 3 shows the cumulated inelastic level densities of the ${ }^{239} \mathrm{Pu}$ target nucleus as a function of excitation energy, $E_{\text {exc }}$. Our calculation (solid curve) exhibits a similar shape but is different in magnitude than the HF-BCS mass model prediction of Goriely [12] (dashed curve) available from the RIPL-2 database. Similar cumulated level densities, but at barrier deformations, are shown Fig. 4. The inner barrier calculations disagree in magnitude (thick solid curve compared to the thick dashed curve). However, the HF-BCS higher calculated level density might be compensated by a higher inner barrier value (Table 1 ). The outer barrier level densities agree in magnitude above $3.75 \mathrm{MeV}$ (thin solid curve compared to the thin dashed curve), but the HF-BCS outer barrier value, smaller than the HF-BCS inner value, is still higher than our value (Table 1). Above the individual level density matching energy, our combinatorial level density calculation has been tuned to obtain a better agreement between our calculated fission cross section and the ENDF/B-VII.0 evaluation. A convenient approach based on the breaking of the level density into energy regions (or phases), $\phi$, of simple exponential increase, each characterized by a numerical constant $\left(C_{\phi}\right)$, a temperature $\left(T_{\phi}\right)$ and a spin-dispersion coefficient $\left(\sigma_{\phi}\right)$, has been adopted:

$$
\rho_{\phi}\left(E_{\text {exc }}\right)=C_{\phi} \frac{(2 J+1)}{4 \sigma_{\phi}^{2}} \exp \frac{\left(-J+\frac{1}{2}\right)^{2}}{2 \sigma_{\phi}^{2}} \exp \left(E_{\text {exc }} / T_{\phi}\right)
$$

with $\sigma_{\phi}^{2}=C_{\phi}^{1}+C_{\phi}^{2} \cdot \sqrt{E}+C^{3} \cdot E$. This explains the nonregular shape changes observed in our calculation (Fig. 4).

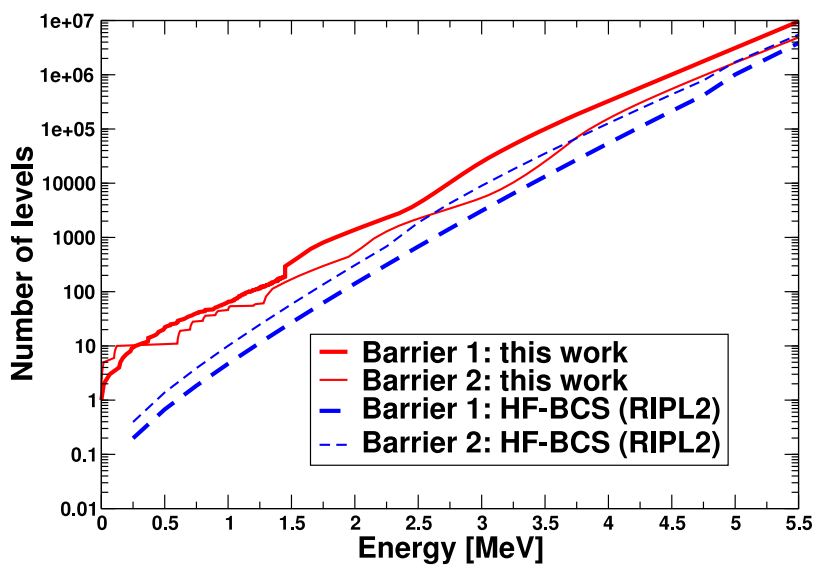

Fig. 4. Cumulated transition states densities of the ${ }^{240} \mathrm{Pu}^{*}$ compound nucleus as a function of the excitation energy. The solid curves correspond to our calculations which are compared to the HF-BCS predictions (dashed-curves) tabulated in the RIPL-2 database. The thick curves are relative to the inner barrier and the thin curves to the outer barrier. At low energies, our calculations are based on individual level sequences which match the continuum level density calculation at 1.5 and $1.3 \mathrm{MeV}$ respectively for the inner and outer barriers.

\section{Treatment of Underlying Intermediate Structure in Fission Cross sections}

\subsection{Above barrier excitation energies}

The presence of the inner barrier introduces a simple decoupling effect between the normal and meta-stable regions of deformation. This decoupling is expressed by two independent fission barrier transmission coefficients $T_{A}$ and $T_{B}$, with barriers commonly chosen as inverted parabolas. The resulting effective transmission coefficient for compound nucleus states of the primary well through specific outer deformation channel $(\mu)$ is simply:

$$
T_{e f f}^{(\mu)}=\frac{T_{A} T_{B(\mu)}}{T_{A}+T_{B}} .
$$

In this strong coupling approximation, $T_{e f f}^{(\mu)}$ is used directlly in the HF formalism as if there was one barrier only. To obtain a more accurate calculation, the fluctuations of the class-I and class-II fission widths (so-called $S_{n f}^{(I)}$ and $S_{n f}^{(I I)}$ ) must be considered assuming a chi-square statistical distribution with an effective number of degrees of freedom different for each barrier. The resulting average fission cross section is:

$$
\begin{aligned}
\sigma_{n f}^{J^{\pi}} & =\sigma_{n,(C N)}^{J^{\pi}} P_{f} \\
& =\pi \lambda^{2}\left(g_{J}\right) T_{I(n)} \sum_{\mu=1}^{\mu_{\text {outer }}^{\text {max }}} \frac{T_{A} T_{B(\mu)}}{T_{A}+T_{B}} S_{n f}^{(I)} S_{n f}^{(I I)} .
\end{aligned}
$$

\subsection{Sub-barrier excitation energies}

Below the barriers, equation (18) is no more valid. Assuming that the coupling between a single class-II state and its class-I neighboring states is still quite strong (moderately weak coupling corresponding to high inner barrier and $\left.\Gamma_{\lambda_{I I}}(f) \sim D_{I I}\right)$, a Lorentzian energy dependence of the fission width can be used to calculate the average fission cross section over a single resonance, with an average over a class-II resonance. Moreover assuming that the statistical fluctuations of widths, spacing and coupling elements can be ignored (so-called "picket fence model"), the resulting average fission probability is:

$$
P_{f}=1+\left(\frac{T_{I}}{T_{(f)}}\right)^{2}+\frac{2 T_{I}}{T_{(f)}} \operatorname{coth}\left(\frac{T_{A}+T_{B}}{2}\right)^{-1 / 2},
$$

with $T_{I}$, the total transmission coefficient over all radiation, elastic and inelastic channels and $T_{(f)}$, the statistical fission transmission coefficient of Eq. 18.

Monte Carlo approach. To circumvent the exact analytical calculation of the fluctuations which is hardly tractable, a Monte Carlo approach, described in Reference [6] can be used. In this approach individual values of all parameters involved in the class-I and II states coupling treatment 
(and identified in section 2.2) are sampled from the appropriate distribution functions and mean values. Preliminary results for ${ }^{239} \mathrm{Pu}$ and ${ }^{240} \mathrm{Pu}$ fission cross sections are shown on Figs. 5 and 6 where the impact of the various approximations on the fission transmission coefficient with respect to the exact Monte Carlo approach can be assessed.

The net effect of the fluctuations can be estimated from the Uniform Intermediate Structure formula without (UIS $\equiv$ Eq. 19; dotted curve) and with (UIS $\times$ fluct.; dotteddashed curve) fluctuation terms. The difference, decreasing with energy, on the average cross section is as much as $30 \%$ depending on the number of fission channels open.

The effect of the intermediate structure (UIS $\times$ fluct. E Eq. 19 multiplied by fluctuation terms; dotted-dashed curve) is to lower the average cross section below the value obtained in the statistical model $(\mathrm{HF} \times$ fluct. $\equiv$ Eq. 18 ; dashed curve). This effect is expected to be larger for subthreshold fission in fertile nuclides $\left({ }^{240} \mathrm{Pu}\right)$ rather than for fissile isotopes $\left({ }^{239} \mathrm{Pu}\right)$.

The exact Monte Carlo treatment (thick solid curve), taking into account both fluctuations and intermediate structures, reproduces quite well the experimental data below $200 \mathrm{keV}$. Above this energy, an additional tuning of fission barrier parameters should be done to improve the agree-

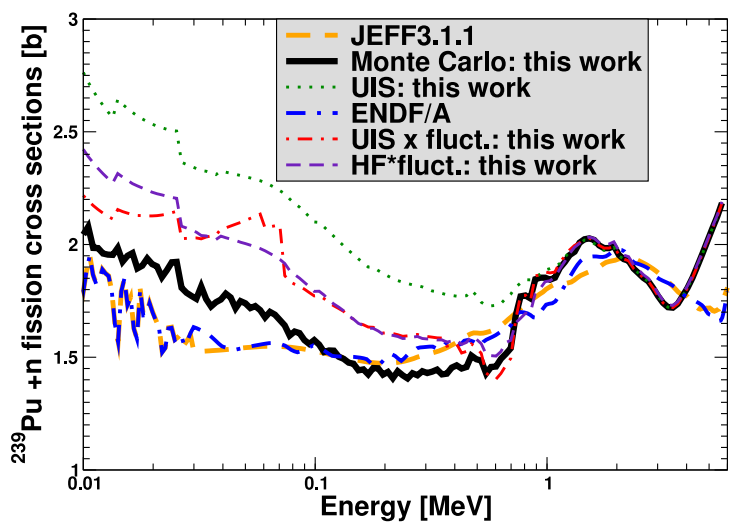

Fig. 5. ${ }^{239} \mathrm{Pu}$ fission cross sections calculated under various approximations (see text for details) and compared to recent evaluated libraries.

\section{Perspectives}

The accurate, physics-based, and predictive modeling of fission cross sections above the resolved resonance range is the ultimate goal of our current efforts. Using BjornholmLynn's theory for the treatment of intermediate structures, model parameters extracted from modern theoretical nuclear structure calculations, and a database of consistent parameters across isotopes, such modeling appears within reach. Our ongoing LANL-CEA collaboration is addressing this topic with promising preliminary results, as shown in this paper. Obviously much work remains to encompass the full complexity (multi-dimensional, time-dependent, temperature-dependent) of the nuclear fission problem.

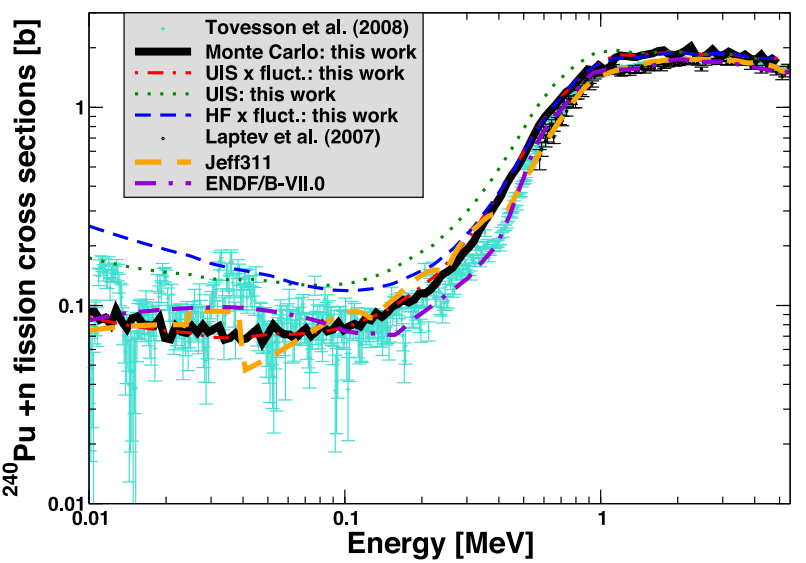

Fig. 6. ${ }^{240} \mathrm{Pu}$ fission cross sections calculated under various approximations (see text for details) and compared to recent evaluated libraries. Recent measurements by Tovesson et al.[15] and Laptev et al. [16] are also shown.

Acknowledgments One of the authors (O. Bouland) expresses his deep gratitude to the T-2 group of LANL for hosting and funding complement. This work would have not been possible without the strong support of the Commissariat à l'Énergie Atomique (Direction de l'Énergie Nucléaire).

This work was carried out under the auspices of the National Nuclear Security Administration of the U. S. Department of Energy at Los Alamos National Laboratory under Contract No. DEAC52-06NA25396.

\section{References}

1. D. Paya et al., J. Phys. 29, (1968) 159.

2. E. Migneco and J.P. Theobald, Nucl. Phys. A112, (1968) 603.

3. J. E. Lynn, J. Phys. A: Math. Nucl. Gen., 6, (1973) 542.

4. G.D. James et al., Phys. Rev. C15 (1977) 2083.

5. H. Weigmann et al., Nucl. Phys. A438 (1985) 333.

6. J. E. Lynn and A. C. Hayes, Proc. of $3^{\text {rd }}$ Intl. Conf. on Fission and Properties of Neutron-Rich Nuclei, (Sanibel Island, FL 2002).

7. A. Bohr, Proc. Intl. Conf. on Peaceful Uses of At. Energies 2 (New York, 1956) 151.

8. F. E. Chukreev and B. Singh, Nucl. Data Sheets 103, (2004) 325-388.

9. R. B. Firestone, Table of Isotopes CD-ROM, $8^{\text {th }}$ edition (Frank Chu Ed., Berkeley, March 1996).

10. S. G. Nilsson et al., Nucl. Phys. A131, (1969) 1.

11. P. Möller et al., Nucl. Phys. A192, (1972) 529.

12. S. Goriely, F. Tondeur, J. M. Pearson, Atomic Data Nuclear Data Tables 77, (2001) 311.

13. P. Möller et al., Phys. Rev. C 79,(2009) 064304.

14. J. E. Lynn, Harwell Report, AERE-R7468, (1974).

15. F. Tovesson et al., Phys. Rev. C 79, 014613 (2009).

16. A. Laptev et al., Proc. of $3^{\text {rd }}$ Intl. Conf. on Fission and Properties of Neutron-Rich Nuclei, (Sanibel Island, FL, 2002). 